\title{
Positive transcriptional control of the pyridoxal phosphate biosynthesis genes $p d x S T$ by the MocR-type regulator PdxR of Corynebacterium glutamicum ATCC 13032
}

Correspondence

Andreas Tauch

tauch@cebitec.uni-bielefeld.de

Received 20 August 2010

Accepted 11 September 2010

\author{
Nina Jochmann, ${ }^{1,2}$ Susanne Götker ${ }^{1}$ and Andreas Tauch ${ }^{1}$ \\ ${ }^{1}$ Institut für Genomforschung und Systembiologie, Centrum für Biotechnologie, Universität \\ Bielefeld, Universitätsstraße 27, D-33615 Bielefeld, Germany \\ ${ }^{2}$ International NRW Graduate School in Bioinformatics and Genome Research, Centrum für \\ Biotechnologie, Universität Bielefeld, Universitätsstraße 25, D-33615 Bielefeld, Germany
}

The $p d x R$ (cg0897) gene of Corynebacterium glutamicum ATCC 13032 encodes a regulatory protein belonging to the MocR subfamily of GntR-type transcription regulators and consisting of an amino-terminal winged helix-turn-helix DNA-binding domain and a carboxy-terminal aminotransferase-like domain. A defined deletion in the $p d x R$ gene resulted in the decreased expression of the divergently orientated $p d x S T$ genes coding for the subunits of pyridoxal 5 '-phosphate synthase. The $p d x S T$ mutant $C$. glutamicum NJ0898 and the $p d x R$ mutant $C$. glutamicum $\mathrm{AMH} 17$ showed vitamin $\mathrm{B}_{6}$ auxotrophy that was restored by supplementing the growth medium with either pyridoxal, pyridoxal 5 '-phosphate or pyridoxamine. The genetic organization of the $89 \mathrm{bp} p d x R-p d x S T$ intergenic region was elucidated by mapping the $5^{\prime}$ ends of the respective transcripts, followed by detection of typical promoter sequences. Bioinformatic pattern searches and comparative genomics revealed three DNA motifs with the consensus sequence AAAGTGGW(-/T)CTA, overlapping the deduced promoter sequences and serving as candidate DNA-binding sites for PdxR. DNA band shift assays with the purified PdxR protein demonstrated the specific binding of the transcription regulator to double-stranded 40-mer sequences containing the detected motifs, thereby confirming the direct regulatory role of $\mathrm{PdxR}$ in activating the expression of the $p d x S T$ genes.

\section{INTRODUCTION}

The term vitamin $\mathrm{B}_{6}$ collectively refers to the three vitamers pyridoxine, pyridoxamine and pyridoxal as well as to their respective $5^{\prime}$-phosphorylated forms (Eliot \& Kirsch, 2004). The vitamin is an essential metabolite in all organisms and is required as a cofactor for numerous enzymes catalysing a wide variety of biochemical reactions, predominantly in amino acid metabolism. Pyridoxal $5^{\prime}$-phosphate (PLP) is the major biochemically active form of vitamin $\mathrm{B}_{6}$ (Eliot \& Kirsch, 2004). Two mutually exclusive pathways for the de novo biosynthesis of vitamin $\mathrm{B}_{6}$ exist in bacteria, referred to as deoxyxylulose 5-phosphate (DXP)-dependent and DXP-independent (Fitzpatrick et al., 2007). The DXPdependent pathway is restricted, with a few exceptions, to the gammaproteobacteria, whereas the DXP-independent

\footnotetext{
Abbreviations: Cy3, indocarbocyanine; DXP, deoxyxylulose 5-phosphate; E-b/O, effector-binding and/or oligomerization; IMPACT, intein mediated purification with an affinity chitin-binding tag; PLP, pyridoxal 5'-phosphate; RACE, rapid amplification of cDNA ends; SOEing, splicing by overlap extension.
}

pathway is much more predominant and is found in most eubacteria (Tanaka et al., 2005). The key enzyme of the DXP-independent pathway is PLP synthase, consisting of the synthase subunit $\mathrm{PdxS}$ and the glutaminase subunit PdxT (Strohmeier et al., 2006). PLP synthase catalyses the direct formation of PLP from pentose sugars (ribose 5phosphate or ribulose 5-phosphate) and triose sugars (glyceraldehyde 3-phosphate or dihydroxyacetone phosphate) in addition to glutamine (Fitzpatrick et al., 2007). Although the enzymic reaction of PLP synthase is of fundamental importance for bacterial metabolism, the transcriptional regulation of the $p d x S$ and $p d x T$ genes has not been investigated so far, but is addressed for the first time in the present study.

During our work on the systematic reconstruction of the transcriptional regulatory network in the Gram-positive model organism Corynebacterium glutamicum (Brinkrolf et al., 2007; Kohl \& Tauch, 2009), we recognized the $\mathrm{Cg} 0897$ (PdxR) protein as a candidate to control the expression of the $p d x S T$ genes in this species. According to genome annotation data, the Cg0897 protein was grouped 
into the GntR family of DNA-binding transcription regulators (Brune et al., 2005). Inactivation of the cg0897 $(p d x R)$ gene by either random transposon mutagenesis with an IS6100-based mobile element (Mormann et al., 2006) or directed gene replacement (McHardy et al., 2003) resulted in vitamin $\mathrm{B}_{6}$ auxotrophy of the respective mutant strains and indicated that the $p d x S T$ genes of C. glutamicum (cg0898 and cg0899) are probably under positive transcriptional control by PdxR. Furthermore, the level of $p d x R$ gene expression was enhanced during the response of $C$. glutamicum to severe heat shock conditions (Barreiro et al., 2009), whereas the expression of the $p d x S T$ genes was slightly down-regulated in the lexA mutant C. glutamicum NJ2114 (Jochmann et al., 2009). In the latter study, an SOS box was detected upstream of the $p d x S T$ gene region and the specific binding of the SOS response regulator LexA was demonstrated in vitro by DNA band shift assays, integrating the $p d x S T$ genes into the SOS regulon of C. glutamicum.

In the present study, we analyse the transcriptional regulation of the PLP synthase genes $p d x S T$. We measure the expression of the $p d x S T$ genes in the wild-type strain and in a $p d x R$ mutant in response to the presence of the $\mathrm{B}_{6}$ vitamers and search for promoter sequences and candidate DNA-binding sites by a bioinformatic approach. The direct interaction of the purified PdxR protein with the candidate DNA-binding sites is shown in vitro by DNA band shift assays.

\section{METHODS}

Bacterial strains, plasmids and growth conditions. Bacterial strains and plasmids used in this study are listed in Table 1. Escherichia coli $\mathrm{DH} 5 \alpha \mathrm{MCR}$ was grown at $37^{\circ} \mathrm{C}$ in Luria-Bertani (LB) medium (Sambrook et al., 1989) and used for standard cloning procedures. All C. glutamicum strains were routinely grown at $30{ }^{\circ} \mathrm{C}$ in LB medium or in minimal medium CGXII containing (per litre) $30 \mu$ g protocatechuic acid and $420 \mu \mathrm{g}$ thiamin (Keilhauer et al., 1993). To analyse the nutritional requirement of C. glutamicum NJ0898 and C. glutamicum AMH17, the CGXII medium was supplemented with $20 \mu \mathrm{M}$ of either pyridoxal, PLP, pyridoxamine or pyridoxine. Growth of C. glutamicum strains was monitored at time intervals of $15 \mathrm{~min}$ by nephelometry by using a Nephelostar Galaxy nephelometer (BMG Laboratories). Antibiotics for plasmid selection were ampicillin $\left(200 \mu \mathrm{g} \mathrm{ml}^{-1}\right.$ for $E$. coli) and kanamycin $\left(50 \mu \mathrm{g} \mathrm{m} l^{-1}\right.$ for $E$. coli and $25 \mu \mathrm{g} \mathrm{ml}^{-1}$ for $C$. glutamicum). Growth of E. coli cultures was monitored by measuring $\mathrm{OD}_{600}$ with an Eppendorf BioPhotometer.

DNA techniques. Preparation of plasmid DNA from E. coli cells by the alkaline lysis technique was performed by using a QIAprep Spin Miniprep kit (Qiagen). Chromosomal DNA of C. glutamicum was prepared as described previously (Tauch et al., 1995). Restriction of DNA, analysis by agarose gel electrophoresis and DNA ligation were performed according to standard protocols (Sambrook et al., 1989). DNA transfer was carried out by electroporation with competent $E$. coli and C. glutamicum cells (Tauch et al., 1994, 2002). DNA amplification by PCR was performed with a PTC-100 thermocycler (MJ Research) and Taq DNA polymerase (Bioline) or Phusion DNA polymerase (Finnzymes). All PCR products were purified with a PCR Purification Spin kit (Qiagen).

Construction of the pdxST mutant C. glutamicum NJ0898. The gene SOEing (gene splicing by overlap extension) procedure (Horton et al., 1989) was used to construct a $p d x S T$ mutant in C. glutamicum ATCC 13032. The PCR primers used were: pdxSTdell (5'-GATCTAGGATCCGCAACCACTCGTGTGAAG), pdxSTdel2 (5'-TTCGGACTGCTCGATGAA), pdxSTdel3 (5' -TTCATCGACGAGCCGAACGCTGGCCTGATCTATCT) and pdxSTdel4 (5'-GATCTAGCATGCCTTGGTGGATGCGGTAAT). The gene SOEing product was digested with BamHI and SphI and subsequently cloned in E. coli DH5 $\alpha \mathrm{MCR}$ into the vector pK18mobsacB (Table 1). The resulting derivative pK18mobsacB_ $\Delta p d x S T$ was suitable for performing an allelic exchange by homologous recombination in the chromosome of C. glutamicum ATCC 13032 (Schäfer et al., 1994), resulting in the mutant strain C. glutamicum NJ0898.

Complementation of the pdxR mutant C. glutamicum AMH17. The complete $p d x R$ ( $c g 0897)$ gene was amplified from chromosomal DNA of C. glutamicum ATCC 13032 by PCR with the primer pair compl_A (5'-GATCTAGAGCTCGATGATCCAACGCCACAA) and compl_B (5'-GATCTACTGCAGCTCTCGGGCTGTTTTTTC). The PCR product was digested with $S a c$ I and PstI and subsequently cloned in E. coli DH5 $\alpha \mathrm{MCR}$ into the vector pEC-K18mob2 (Table 1). The resulting plasmid pEC-K18mob2_pdxR was transferred to the mutant strain C. glutamicum AMH17 by electroporation.

Table 1. Bacterial strains and plasmids used in this study

\begin{tabular}{|c|c|c|}
\hline Strain or plasmid & Relevant characteristics & Source or reference \\
\hline C. glutamicum ATCC 13032 & Wild-type strain & ATCC \\
\hline C. glutamicum АMH17 & Restriction-deficient strain with defined deletion in $p d x R$ & McHardy et al. (2003) \\
\hline C. glutamicum NJ0898 & Wild-type strain with defined deletion in $p d x S T$ & This study \\
\hline E. coli $\mathrm{DH} 5 \alpha \mathrm{MCR}$ & E. coli strain used for standard cloning procedures & Grant et al. (1990) \\
\hline E. coli ER2566 & E. coli strain used for heterologous gene expression & New England Biolabs \\
\hline E. coli $\mathrm{TOP} 10$ & E. coli strain used for cloning of RACE-PCR products & Invitrogen \\
\hline pK18mobsacB & sacB lacZ $\alpha \mathrm{Km}^{\mathrm{r}}$; cloning vector for allelic exchange & Schäfer et al. (1994) \\
\hline pK18mobsacB_spdxST & pK18mobsacB carrying a modified $p d x S T$ gene region & This study \\
\hline pEC-K18mob2 & $\mathrm{Km}^{\mathrm{r}}$; E. coli/C. glutamicum cloning vector & Kirchner \& Tauch (2003) \\
\hline pEC-K18mob2_pdxR & pEC-K18mob2 carrying the C. glutamicum $p d x R$ gene & This study \\
\hline pTXB1 & $\mathrm{Ap}^{\mathrm{r}} ;$ E. coli vector for protein tagging with an intein/chitin-binding domain & New England Biolabs \\
\hline pTXB1_pdxR & pTXB1 carrying the C. glutamicum $p d x R$ gene & This study \\
\hline pCR2.1-TOPO & $\mathrm{Ap}^{\mathrm{r}} \mathrm{Km}^{\mathrm{r}}$; cloning vector for PCR products & Invitrogen \\
\hline
\end{tabular}


RNA techniques. Preparation of total RNA from C. glutamicum cultures and hybridization of whole-genome DNA microarrays were carried out as described previously (Brune et al., 2007; Jochmann et al., 2009). Differential gene expression was measured by real-time RT-PCR with an OPTICON Continuous Fluorescence Detector (MJ Research) and SensiMix One-Step kit (Quantace). All measurements were performed with RNA probes from two biological replicates, each represented by two technical replicates. The amount of specific transcripts was normalized with respect to total RNA and the relative changes in the transcription rates were determined as $2^{-\Delta \mathrm{CP}}$, where $\Delta \mathrm{CP}$ is equal to the difference of the measured crossing points for the test and control condition. The $5^{\prime}$ ends of transcripts were determined by using the second generation $5^{\prime} / 3^{\prime}$ rapid amplification of cDNA ends (RACE) kit (Roche Diagnostics) according to the manufacturer's instructions. The resulting PCR products were cloned into the pCR2.1-TOPO vector and transferred to chemically competent E. coli TOP10 cells. Sequencing of cloned DNA fragments was performed by IIT Biotech.

Expression and purification of the C. glutamicum PdxR protein. The PdxR protein was purified by using the intein mediated purification with an affinity chitin-binding tag (IMPACT) system (New England BioLabs). The $p d x R$ coding region was amplified by PCR with the primer pair intein_A (5'-GGTGGTCATATGATGCTTGCCGACCTTCCC) and intein_B (5'-GGTGGTTGCTCTTCCGCAGGCGCCTAGAGACACCGCATC), adding in this way an alanine residue (underlined) to the carboxy-terminal end of $\mathrm{PdxR}$ to ameliorate cleavage of the intein tag from the recombinant protein. The PCR product was digested with NdeI and SapI and subsequently cloned into the expression vector pTXB1, thereby fusing the recombinant $\mathrm{C}$ terminus of $\mathrm{PdxR}$ to a self-cleavable intein tag that contains a chitin-binding domain. The resulting plasmid pTXB1_ $p d x R$ was transferred to competent E. coli ER2566 cells to overexpress the PdxR protein. E. coli ER2566 cultures were grown overnight at room temperature in $500 \mathrm{ml} \mathrm{LB}$ medium containing $200 \mu \mathrm{g}$ ampicillin $\mathrm{ml}^{-1}$. Induction of $p d x R$ expression was carried out at an $\mathrm{OD}_{600}$ of 0.5 by adding IPTG to a final concentration of $0.5 \mathrm{mM}$. Cells were harvested after $16 \mathrm{~h}$ incubation by centrifugation at $5000 \mathrm{~g}$ and $4{ }^{\circ} \mathrm{C}$ for $15 \mathrm{~min}$. The cell pellet was resuspended in lysis buffer [20 mM Na $2 \mathrm{HPO}_{4}, 500 \mathrm{mM} \mathrm{NaCl}, 1 \mathrm{mM}$ EDTA, $0.1 \%$ (w/v) Triton X-100, $20 \mu \mathrm{M}$ PMSF, $0.5 \mathrm{mM}$ Tris(2-carboxyethyl)phosphine]. Cells were disrupted twice in a FRENCH Pressure Cell (Thermo Scientific) and the cell extract was cleared by centrifugation at $4{ }^{\circ} \mathrm{C}$ for $20 \mathrm{~min}$. The PdxR protein was purified from the protein crude extract by means of the chitin-binding domain of the intein tag, applying a Protino Column (14 ml; Macherey-Nagel) that was packed with chitin beads (New England Biolabs). On-column cleavage was induced by loading the column with cleavage buffer containing $50 \mathrm{mM}$ DTT, and incubating the assay at $4{ }^{\circ} \mathrm{C}$ for $16 \mathrm{~h}$. The recombinant PdxR protein devoid of the intein tag was eluted with column buffer and stored at $-20{ }^{\circ} \mathrm{C}$. The protein concentration was determined with a Bio-Rad protein assay kit. The eluate was analysed by SDS-PAGE, and in-gel digestion with modified trypsin (Promega) was carried out to verify the purity of the PdxR protein. The peptide mass fingerprint of the purified PdxR protein was generated by MALDI-TOF MS, by using an Ultraflex mass spectrometer (Bruker Daltonics) and the MASCOT software. The LexA protein from $C$. glutamicum ATCC 13032 was purified as described previously (Jochmann et al., 2009).

DNA band shift assays with purified PdxR protein. DNA band shift assays were performed with indocarbocyanine (Cy3)-labelled fragments generated by PCR with the primer pair shift_A (5'-Cy3CGAGACGACGGATCTGTT) and shift_B (CGAGTGGTTGCTTGGTAA) or with 40-mers that were annealed with complementary oligonucleotides to double-stranded DNA fragments by incubation at $94{ }^{\circ} \mathrm{C}$ for $5 \mathrm{~min}$ and subsequent cooling to $8{ }^{\circ} \mathrm{C}$ in steps of $1{ }^{\circ} \mathrm{C}$ per
$10 \mathrm{~s}$. The assays were performed in a final volume of $20 \mu \mathrm{l}$ containing 0.05 pmol of the PCR product or double-stranded 40-mers, $40 \mathrm{pmol}$ of the purified protein, $0.06 \mu \mathrm{g}$ herring sperm DNA, $20 \%(\mathrm{v} / \mathrm{v})$ glycerol and binding buffer [20 mM HEPES, $30 \mathrm{mM} \mathrm{KCl}, 10 \mathrm{mM}$ $\left(\mathrm{NH}_{4}\right)_{2} \mathrm{SO}_{4}, 1 \mathrm{mM}$ EDTA, $1 \mathrm{mM}$ DTT, $0.2 \%$ (w/v) Tween 20; $\mathrm{pH}$ 7.6]. The binding buffer was modified where appropriate by adding either 2 or $200 \mu \mathrm{M}$ of the vitamers pyridoxal, PLP, pyridoxamine or pyridoxine. The assays were incubated at $30{ }^{\circ} \mathrm{C}$ for $30 \mathrm{~min}$ and separated in $2 \%$ agarose gels prepared in gel buffer $(40 \mathrm{mM}$ Tris/ $\mathrm{HCl}$, $10 \mathrm{mM}$ sodium acetate, $1 \mathrm{mM}$ EDTA; $\mathrm{pH} 7.8$ ); $70 \mathrm{~V}$ was applied for $1 \mathrm{~h}$. The agarose gels were scanned with a Typhoon 8600 Variable Mode Imager (Amersham Biosciences Europe).

\section{RESULTS}

\section{Molecular features of the PdxR protein from C. glutamicum ATCC 13032}

The PdxR protein (Cg0897) of C. glutamicum ATCC 13032 has a predicted size of 453 amino acids and a theoretical molecular mass of $48.9 \mathrm{kDa}$ (Kalinowski et al., 2003). Protein domain predictions performed with the SUPERFAMILY tools (Gough et al., 2001) and by Conserved Domain Database searches (Marchler-Bauer et al., 2009) revealed that the PdxR protein consists of an aminoterminal winged helix-turn-helix DNA-binding domain of the GntR family of transcription regulators and a carboxyterminal domain belonging to the aspartate aminotransferase superfamily. This fusion of domains in DNA-binding proteins is characteristic of the MocR subfamily of GntRtype transcription regulators (Hoskisson \& Rigali, 2009; Rigali et al., 2002). Aspartate aminotransferases are part of a large superfamily of PLP-binding enzymes (Schneider et al., 2000) sharing a prominent mechanistic feature in such a way that the PLP group is bound covalently to a conserved lysine residue (Sigrist et al., 2010). According to comparative genomics performed with the Integrated Microbial Genomes system (Markowitz et al., 2006), proteins similar to $\mathrm{PdxR}$ from C. glutamicum are apparently encoded upstream of genes encoding subunits of PLP synthase in 12 other actinobacterial genomes. The PdxR protein from C. glutamicum ATCC 13032 showed $37-57 \%$ amino acid sequence identity with the putative PdxR proteins from other actinobacteria (Fig. 1a). An amino acid sequence alignment of the PLP attachment sites present in the predicted aminotransferase domains of the PdxR-like proteins demonstrated that the lysine residue necessary for the covalent binding of PLP is only present in the respective protein from Kocuria rhizophila DC2201 (Fig. 1b). In the other PdxR-like proteins, the lysine residue is replaced by the amino acid serine or alternatively by a threonine or histidine residue. This result suggests that the aminotransferase domain of the actinobacterial PdxR-like proteins is probably unable to bind PLP covalently. At least in the case of C. glutamicum ATCC 13032, this indication fits the previous observation that no aminotransferase activity was detectable in enzyme assays with purified PdxR protein (Marienhagen et al., 2005). 
(a)

Transcription regulator GntR family

\section{C. glutamicum ATCC 13032 \\ [ID\%] \\ 100}

Synthase subunit synthase

Synthase subunit Glutaminase subunit
C. aurimucosum ATCC 700975

C. diphtheriae NCTC 13129

C. genitalium ATCC 33030

C. lipophiloflavum DSM 44291

C. matruchotii ATCC 14266

C. pseudogenitalium ATCC 33035

C. striatum ATCC 6940

C. tuberculostearicum SK141

Brevibacterium lines $B L 2$

Jonesia denitrificans DSM 20603

Propionibacterium acnes KPA171202

Kocuria rhizophila DC2201

$\begin{array}{ll}\text { cauri_0519 } & 55 \\ \text { dip0226 } & 57 \\ \text { HMPREF0291_0673 } & 39 \\ \text { HMPREF0298_1597 } & 45 \\ \text { CORMA0001_0048 } & 55 \\ \text { HMPREF0305_0385 } & 57 \\ \text { HMPREF0308_1600 } & 54 \\ \text { CORTU0001_1288 } & 54 \\ \text { BlinB01003387 } & 37 \\ \text { Jden_1599 } & 39 \\ \text { PPA0964 } & 53 \\ \text { KRH_01150 } & 41\end{array}$

$p d x R$ cg0898

[ID\%] [ID\%]

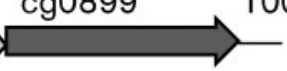

$\begin{array}{llll}\text { cauri } 0520 & 87 & \text { cauri_0521 } & 48 \\ \text { dip } 0 \overline{2} 27 & 91 & \text { dip0 } 228 & 60\end{array}$

HMPREF0291 067474

HMPREF0298 159880

CORMA0001 $0047 \quad 79$

HMPREF0305_0384 88

HMPREF0308-160189

CORTU0001 $1287 \quad 89$

BlinB010033 $\overline{8} 8$

Jden_1600

PPA0̄965

KRH_01130

- HMPREFO298 15994

HMPREF0305 038364

HMPREF0308 ${ }^{-} 160262$

CORTU0001 128656

BlinB01003389 46

Jden 1601

PPĀ̄966

KRH_01120 48

$p d x S$

$p d x T$

(b)

C. glutamicum ATCC 13032

C. aurimucosum ATCC 700975

C. diphtheriae NCTC 13129

C. genitalium ATCC 33030

C. lipophiloflavum DSM 44291

C. matruchotii ATCC 14266

C. pseudogenitalium ATCC 33035

C. striatum ATCC 6940

C. tuberculostearicum SK141

Brevibacterium linens BL2

Jonesia denitrificans DSM 20603

Propionibacterium acnes KPA171202

Kocuria rhizophila DC2201
PLP attachment site

DRTILLGTFSSVITPOVACGYLIA EHTVLLGTFS SVISPSIACGYLVV QRTALLGTFS SVISPQVSCGYIVA ADVFTLGTFS LLLSRNLSAGYVVT ASVIALGTFSILLTRQLSAGYVVA DHTVLLGTFS SVLSPALACGYIVA EHTVLLGTFSSVISPSIACGYLVV EHTVLLGTFSSVISPSIACGYLVV EHTVLLGTFS SVISPSIACGYLVV

AQVVHIGTFSILLTSAVSTGYIIA DQVVHVGTFRHVLSEHVRLGYVIA EHTILLGTFSSVLTPALGCGYLVV DTVVTLGSFA RVLGSSVGVGHLVA . $\quad$ * $^{*}$

(c)

C. glutamicum

C. jeikeium

C. kroppenstedtii

C. urealyticum

C. efficiens

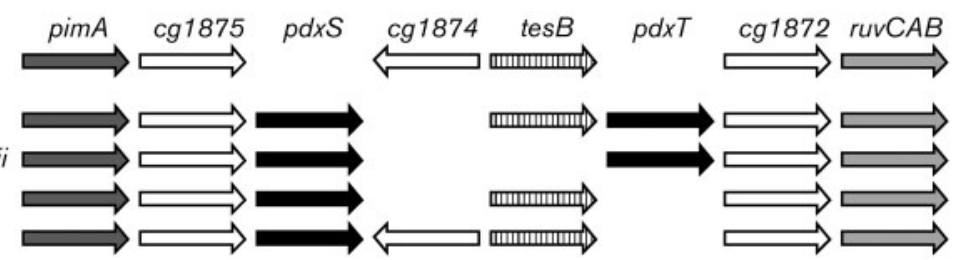

Fig. 1. Comparison of actinobacterial gene regions encoding the transcription regulator PdxR and PLP synthase. (a) Schematic representation of the $p d x R-p d x S T$ gene region in the genomes of 13 actinobacteria. Genes are listed with their GenBank accession nos. The percentage identities [ID\%] of the deduced proteins to the homologous counterparts from C. glutamicum ATCC 13032 are listed. The additional gene $\left(K R H \_01140\right)$ located between $p d x R$ and $p d x S T$ in the $K$. rhizophila genome is indicated by a triangle. (b) Amino acid sequence alignment of PLP attachment sites detected in the aminotransferase-like domain of actinobacterial PdxR transcription regulators. The position of the conserved lysine residue generally used for the covalent binding of PLP is boxed. It is missing in the actinobacterial PdxR-like regulators, with the exception of the K. rhizophila protein $\mathrm{KRH}$ _01150. The amino acid sequence alignment was generated with the CLUSTAL W tool (Larkin et al., 2007). (c) Schematic representation of the $p d x S$ locus in corynebacterial genomes lacking a $p d x R$-like regulatory gene. Homologous genes share the same shading. The gene regions from C. glutamicum ATCC 13032 are shown for comparison.

The $p d x R$ gene of C. glutamicum ATCC 13032 is located in a head-to-head arrangement with the $p d x S T$ genes (Fig. 1a). This divergent order of $p d x R$ and $p d x S T$ genes is conserved in 13 actinobacterial genomes encoding PdxRlike proteins, with the exception of 'Corynebacterium genitalium' ATCC 33030 and Corynebacterium matruchotii ATCC $14266^{\mathrm{T}}$, both of which lack a $p d x T$ gene downstream of $p d x S$ (Fig. 1a). Another exception was observed in the genome of $K$. rhizophila DC2201, which includes an additional gene between the $p d x R$ and $p d x S T$ coding regions. It is interesting to note that the $p d x R-p d x S T$ gene clusters are located in different regions of the respective 13 actinobacterial genomes (Markowitz et al., 2006). When comparing the mean $\mathrm{G}+\mathrm{C}$ content of all coding regions of 
the C. glutamicum ATCC 13032 genome (54.8 mol\%) with that of the $p d x R$ (64.5 mol\%), $p d x S(60.9 \mathrm{~mol} \%)$ and $p d x T$ (57.4 mol\%) genes, a deviation in the DNA base composition was detected, i.e. $p d x R$ and $p d x S T$ are characterized by a significantly higher DNA G + C content. A comparative genomics approach with the Integrated Microbial Genomes system (Markowitz et al., 2006) revealed that the $p d x S(T)$ genes from other corynebacteria that lack a $p d x R$-like regulatory gene, such as Corynebacterium jeikeium, Corynebacterium kroppenstedtii, Corynebacterium urealyticum and Corynebacterium efficiens, are located in a conserved region of the genome close to the ruv $C A B$ operon (Fig. 1c). The corresponding gene locus is also present in the genome of C. glutamicum ATCC 13032, which, however, lacks the $p d x S T$ genes (Fig. 1c). The biosynthesis pathway for vitamin $B_{6}$ has been shown to be very dynamic with respect to evolutionary changes (Tanaka et al., 2005), and a putative genetic replacement of $p d x S T$ by PdxR-regulated counterparts in C. glutamicum might be another example of gene gain and gene loss in this part of bacterial metabolism.

\section{Deletion of the PLP biosynthesis genes pdxST}

The PdxS and PdxT proteins represent the subunits of PLP synthase, which catalyses the de novo biosynthesis of PLP via the DXP-independent pathway (Fitzpatrick et al., 2007). The PdxS protein from C. glutamicum ATCC 13032 showed 74$91 \%$ amino acid sequence identity with predicted PdxS proteins from other actinobacteria, whereas the range of sequence identity of PdxT proteins was $44-64 \%$ (Fig. 1a). To analyse the physiological role of the PLP synthase genes in $C$. glutamicum ATCC 13032, a pdxST mutant was constructed by introducing a marker-free deletion into the $p d x S T$ gene region (Table 1). The resulting mutant C. glutamicum NJ0898 was subsequently characterized by growth assays in CGXII minimal medium supplemented with the vitamers pyridoxal, PLP, pyridoxamine or pyridoxine (Fig. 2a). Deletion of the pdxST genes in C. glutamicum NJ0898 resulted in vitamin $\mathrm{B}_{6}$ auxotrophy, which was restored by external pyridoxal, PLP or pyridoxamine (Fig. 2a). Growth of C. glutamicum NJ0898 in the presence of pyridoxal or PLP was indistinguishable from that of a wild-type control, whereas supplementing of CGXII medium with pyridoxamine led to a prolonged lag phase of the culture. No growth of C. glutamicum NJ0898 was observed when the medium was supplemented with the vitamer pyridoxine (Fig. 2a). This suggests that the $p d x S T$ genes are essential for the biosynthesis of PLP in C. glutamicum ATCC 13032. Moreover, the successful supplementation of the mutant strain with different vitamers indicates that a vitamin $B_{6}$ salvage pathway for the utilization of pyridoxal and pyridoxamine is functional in C. glutamicum ATCC 13032 (Fitzpatrick et al., 2007). In this pathway, the vitamers can be phosphorylated through the action of the specific kinase PdxK. A corresponding gene encoding PdxK (cg1041) was predicted in the genome sequence of C. glutamicum ATCC 13032 (Ikeda \& Nakagawa, 2003; Kalinowski et al., 2003).
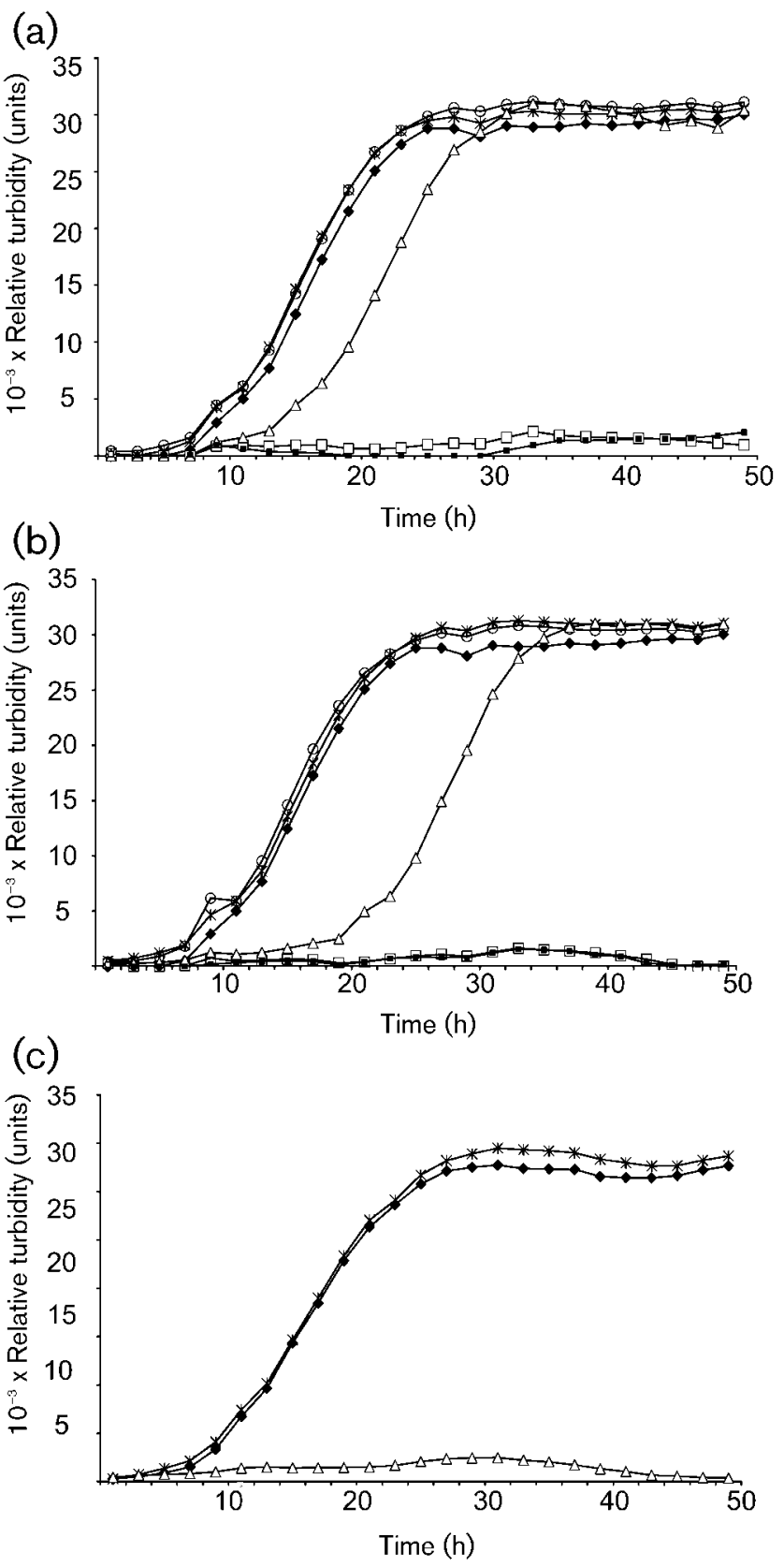

Fig. 2. Phenotypic characterization of C. glutamicum NJ0898 and C. glutamicum AMH17. Growth in CGXII minimal medium was monitored by nephelometry and is shown as relative turbidity. Values are the means of measurements of six biological replicates. (a) Growth of the pdxST mutant C. glutamicum NJ0898 and the wild-type strain C. glutamicum ATCC 13032. (b) Growth of the $p d x R$ mutant $C$. glutamicum $\mathrm{AMH} 17$ and the wild-type control ATCC 13032. In (a) and (b): $>$, ATCC 13032; $\square$, NJ0898/ AMH1 7 ; $\times$, plus pyridoxal; $\bigcirc$, plus PLP; $\triangle$, plus pyridoxamine; plus pyridoxine. (c) Complementation of the $p d x R$ mutant $C$. glutamicum AMH17. Plasmid pEC-K18mob2 $\_d x R$ contains the $p d x R$ gene for complementation ( $\times$ ). C. glutamicum ATCC 13032 carrying the empty cloning vector $\mathrm{pEC}-\mathrm{K} 18$ mob2 served as a control $(\triangle)$. ATCC 13032 (pEC-K18mob2). 


\section{Differential expression of $p d x$ genes in response to $B_{6}$ vitamers}

Expression of the $p d x S T, p d x K$ and $p d x R$ genes was analysed in response to the presence of the four $B_{6}$ vitamers. The wildtype strain C. glutamicum ATCC 13032 was grown in CGXII minimal medium supplemented with pyridoxal, PLP, pyridoxamine or pyridoxine. Expression of the $p d x$ genes was measured by real-time RT-PCR, by using total RNA probes from exponentially growing cultures. The wild-type strain grown in non-supplemented CGXII medium served as a reference. Expression of $p d x S$ and $p d x T$ was significantly reduced in the presence of pyridoxal or PLP, whereas supplementing with pyridoxamine or pyridoxine showed no effect on $p d x S T$ expression (Fig. 3a). Expression levels of $p d x K$ and $p d x R$ revealed no significant differences from the control assay under any experimental condition (data not shown). This RT-PCR assay suggests that the transcription of the PLP synthase genes $p d x S T$ is controlled in response to the presence of pyridoxal or PLP in C. glutamicum.

\section{Activation of the PLP synthase genes pdxST by PdxR}

The first evidence for a regulatory role of the PdxR protein in the expression of $p d x S T$ was obtained in C. glutamicum by random transposon mutagenesis (Mormann et al., 2006) and by genome-based analysis of aminotransferase genes (McHardy et al., 2003). In the present study, growth of the $p d x R$ mutant C. glutamicum AMH17, containing a defined deletion in the $p d x R$ gene (McHardy et al., 2003), was monitored in CGXII minimal medium supplemented with different vitamers (Fig. 2b). C. glutamicum AMH17 revealed the expected auxotrophy which was restored by supplementing the growth medium with either pyridoxal, PLP or pyridoxamine (Fig. 2b). As observed in the $p d x S T$ mutant $C$. glutamicum NJ0898, growth of C. glutamicum AMH17 was indistinguishable from that of a wild-type control when the CGXII medium was supplemented with pyridoxal or PLP, whereas the addition of pyridoxamine to the medium led to a prolonged lag phase of the culture. As expected, no growth of C. glutamicum AMH17 was observed in the presence of pyridoxine (Fig. 2b). Accordingly, the $p d x R$ mutant $C$. glutamicum AMH17 showed the same growth phenotype as the $p d x S T$ mutant C. glutamicum NJ0898. Moreover, the vitamin $\mathrm{B}_{6}$ auxotrophy of $C$. glutamicum AMH17 was complemented by plasmid pEC-K18mob2_pdxR (Fig. 2c), thereby confirming that the auxotrophic phenotype of this mutant strain was caused by the deletion of the $p d x R$ gene.

To characterize further the phenotype of C. glutamicum AMH17 at the genetic level, expression of $p d x S T$ and $p d x K$ was measured by real-time RT-PCR and compared with a wild-type control. Inactivation of the $p d x R$ gene in $C$. glutamicum AMH17 resulted in decreased expression of $p d x S$ and $p d x T$ (Fig. 4), whereas no difference in $p d x K$ expression was detectable (data not shown). This negative effect of the $p d x R$ mutation on the expression of $p d x S T$ in C. glutamicum AMH17 was complemented by plasmid pEC-K18mob2_pdxR. Expression of the $p d x S$ and $p d x T$ genes remained slightly lower (two- to fourfold) in the complemented C. glutamicum strain when compared with the wild-type control carrying the empty cloning vector (Fig. 4). However, the differential expression pattern of the $p d x S T$ genes in C. glutamicum AMH17 suggested an activating role of the PdxR regulator in PLP biosynthesis.
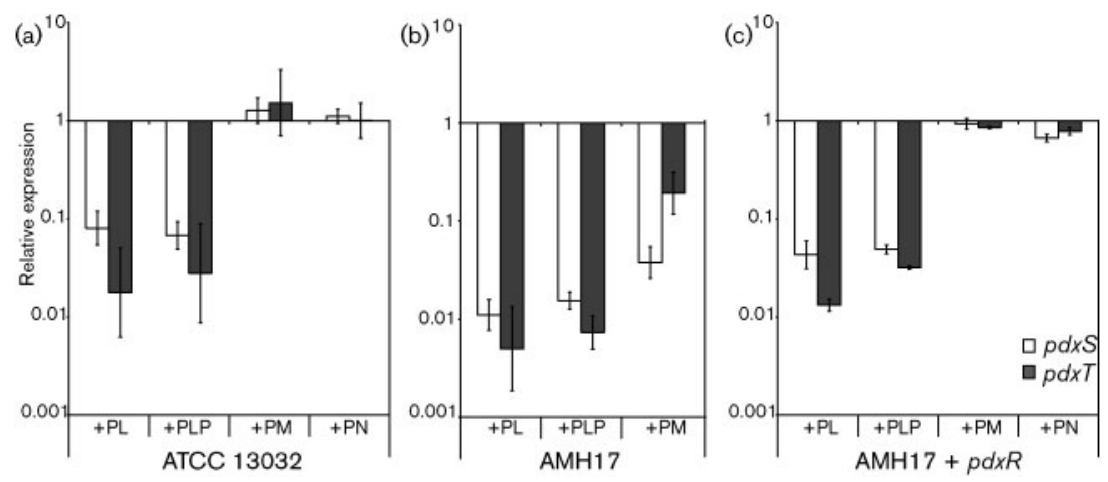

Fig. 3. Relative gene expression of $p d x S$ and $p d x T$ in response to different $B_{6}$ vitamers. Expression levels of $p d x S$ and $p d x T$ were measured by real-time RT-PCR. Values are means $( \pm \mathrm{SD})$ of at least two measurements. The assays were performed with C. glutamicum cultures grown in CGXII medium in the presence of either pyridoxal (PL), pyridoxal 5'-phosphate (PLP), pyridoxamine (PM) or pyridoxine (PN) where appropriate. (a) Relative expression of $p d x S T$ in the wild-type strain C. glutamicum ATCC 13032. Values were normalized by using the measurements determined in C. glutamicum ATCC 13032 grown in nonsupplemented CGXII medium. (b) Relative expression of $p d x S T$ in the $p d x R$ mutant C. glutamicum AMH17. (c) Relative expression of $p d x S T$ in the complemented strain C. glutamicum AMH1 7 (AMH17 $+p d x R$ ). Values in (b) and (c) were normalized by using the measurements determined in C. glutamicum ATCC 13032 carrying the empty cloning vector pEC-K18mob2 and grown in non-supplemented CGXII medium. 


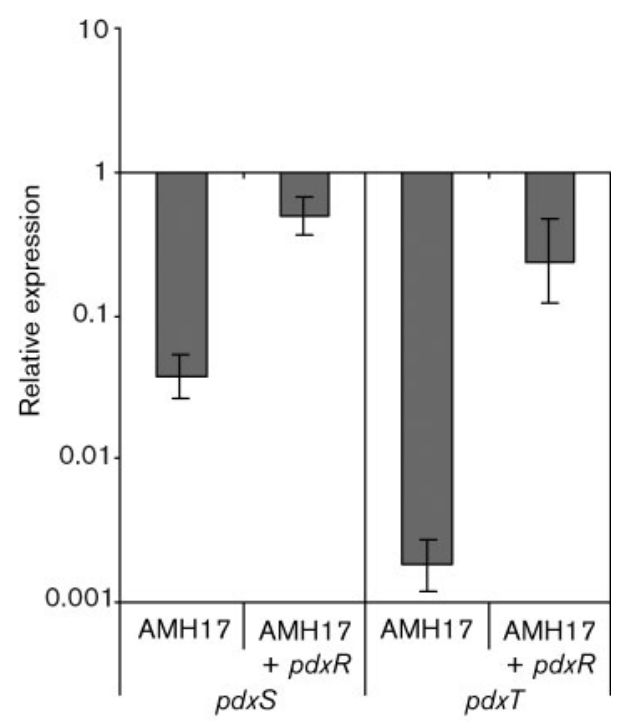

Fig. 4. Complementation of C. glutamicum $\mathrm{AMH} 17$ with the $p d x R$ gene. Relative expression levels of $p d x S$ and $p d x T$ were measured by real-time RT-PCR and normalized by using the values determined in the plasmid-carrying wild-type C. glutamicum ATCC 13032 (pEC-K18mob2). The RNA probes were isolated from C. glutamicum strains grown in LB medium. Values are means $( \pm S D)$ of four measurements. AMH17, C. glutamicum $\mathrm{AMH} 17$ carrying the empty cloning vector pEC-K18mob2; $\mathrm{AMH} 17+p d x R$, C. glutamicum $\mathrm{AMH} 17$ carrying $\mathrm{pEC}-$ K18mob2_pdxR for complementation.

As global gene expression profiling by DNA microarray hybridization revealed no additional target gene with significant changes in gene expression levels (data not shown), PdxR most probably acts as a local activator of $p d x S T$ gene expression in C. glutamicum. According to the divergent orientation of the $p d x R$ and $p d x S T$ genes, this genomic region of C. glutamicum ATCC 13032 represents a local gene regulatory unit commonly referred to as a divergon (Rodionov, 2007).

Furthermore, expression of the $p d x S T$ genes was measured by real-time RT-PCR in the $p d x R$ mutant $C$. glutamicum AMH17 grown in CGXII minimal medium supplemented with pyridoxal, PLP or pyridoxamine (Fig. 3b). As expected, expression of the two genes was reduced under all growth conditions, most probably due to the absence of the $p d x R$ gene product. Expression of $p d x S T$ was also monitored in the complemented mutant strain C. glutamicum AMH17 (Fig. $3 c)$. In this case, expression levels of the $p d x S T$ genes were similar to those of the wild-type strain ATCC 13032 grown in the presence of the corresponding vitamers (Fig. 3a).

\section{Transcriptional organization of the pdxR-pdxST intergenic region}

For a more precise functional analysis of the $89 \mathrm{bp} p d x R-$ $p d x S T$ intergenic region, $p d x R$ and $p d x S T$ promoter sequences and candidate DNA-binding sites of PdxR were predicted (Fig. 5). First, total RNA was isolated from exponentially growing C. glutamicum ATCC 13032 cells and used to amplify the $5^{\prime}$ ends of the $p d x R$ and $p d x S T$ transcripts by RACE-PCR. In both cases, the detected $5^{\prime}$ ends were identical to the adenine residue of the annotated start codons (Fig. 5a), indicating the presence of so-called leaderless transcripts that are known to occur in C. glutamicum (Pátek et al., 2003). The $5^{\prime}$ ends were used to deduce potential -10 and -35 promoter sequences according to the characteristic features of corynebacterial promoters (Pátek et al., 2003). The proposed promoter regions of $p d x R$ (TTGCAT- $\mathrm{N}_{17^{-}}$ GACGAT) and $p d x S T$ (CCGCTA- ${ }_{18}$-GATCAT) revealed weak similarity to the consensus sequence of $C$. glutamicum promoters, with three to four matches to the -10 and -35 hexamer consensus sequences (TTGCCA- $\mathrm{N}_{17 / 18^{-}}$TAYAAT).

The computer program REPuter (Kurtz et al., 2001) was used to detect direct and inverted repeat sequences in the small $p d x R-p d x S T$ intergenic region (Fig. 5a). An 11 bp inverted repeat (motif 1 and motif 3 ) with one mismatch at position 8 and a spacing of $29 \mathrm{bp}$ was found (AAAGTGG[A/T]CTA). Both DNA motifs are located between the mapped -10 and -35 hexamers of the $p d x R$ and $p d x S T$ promoters (Fig. 5a). Interestingly, motif 1 overlaps the previously detected DNA-binding site (SOS box) of the SOS response regulator LexA from $C$. glutamicum (Jochmann et al., 2009). An additional $12 \mathrm{bp}$ sequence, termed motif 2 (AAAGTGGATCTA), showed similarity to motif 1 and motif 3 and was identified between the -35 promoter regions (Fig. 5a). A bioinformatic search for identical DNA motifs in the genome sequence of C. glutamicum ATCC 13032 with the Patscan program (Dsouza et al., 1997) revealed an additional appearance of motif 1 within the coding region of $\operatorname{cg} 1490$ (encoding a putative NTP pyrophosphohydrolase) and of motif 3 within the coding region of $\operatorname{cg} 0570$ (encoding a putative dehydrogenase). Neither gene was detected as being differentially expressed by genome-wide transcriptional profiling of the $p d x R$ mutant C. glutamicum AMH17 (data not shown), supporting the view that the transcription regulator PdxR acts as a local activator of the $p d x S T$ genes.

To provide more evidence for the biological significance of the three motifs, the nucleotide sequences of the $p d x R-$ $p d x S T$ intergenic regions from six corynebacteria, Propionibacterium acnes and Jonesia denitrificans were aligned with the CLUSTAL $\mathrm{W}$ tool (Larkin et al., 2007). This DNA alignment revealed that the direct and inverted repeat sequences represented by motif 1 to motif 3 are conserved in the $p d x R-p d x S T$ intergenic regions of the eight actinobacterial genomes (Fig. 5b). Motif 1 revealed the highest level of DNA sequence conservation, with eight of 11 matching bases. These DNA motifs are therefore likely candidates for DNA-binding sites of the transcription regulator PdxR. As knowledge of DNA-binding sites of MocR-type transcription regulators is very scarce (Rigali et al., 2002), the functional relevance of the three motifs 


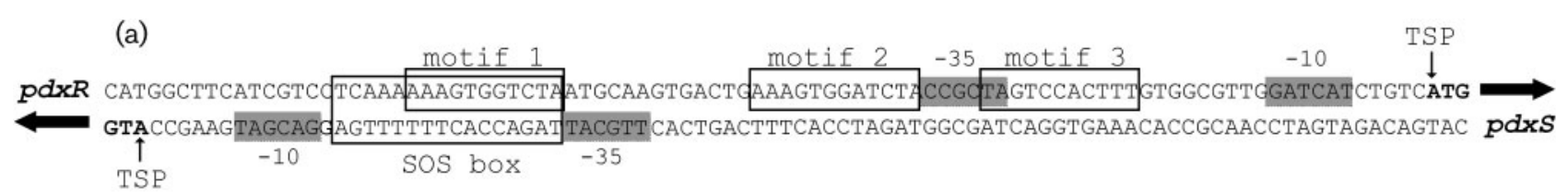

(b)

motif 1 motif 4 motif 2

motif 3

$\mathrm{Pa}$ CATGACGGTGGACCGA------AAAGTGGTCTAACAAAGTGGTCAIATATGGATCTT GTCATGAACCACAATCGTGGATTTC-ATG---------CACGTTGCCTCCCACTCCGCGGAACTGGTCTA GTCAAACTCATCATAACTGGATCTICCAdTGGACCAGCCTGGTGGGTCATGCTTG-GCAACA -------CACGACCCCAT-CATAAGTGGTCTAATTCGACACTCGGAAAATGGCTCTT GTCTTGGACCAATTTTCTGGGCCACTATGTGAGCCATG CATAGAGGTATTGTGATGTGCAAAAGTGGTCTATTGCAACATGCTTAATTTGGATCTAGTGTTAGTCCACTTTTATAGGCTTGGATGTTCACTATG -------CATTGCCCGAAGGTAACTGGTCCAAAGAATCTAAATCAAACTGGCACTAGGAATCTACCAGTG-GGAGGGTGAGACTAGT-GCAATG CATGGTTGTTATCCAACCCGCCAAAGTGGTCTA.GTGCAACTTGTGGTGTTTGGATCTGGTGGTAAACCACTTACGCAGGTTTG-ATAGACCGCATG ------CATGGTCCCAG-CATAAGTGGTCTAATTCAACACGCTTGAATTGGCTCTT GTCTTAGACCATTTOTTAGTGTGAGGATGGTCCGCATG CATGGCTTCATCGTCCTCA-AAAAAGTGGTCTAATGCAAGTGACTGAAAGTGGATCTACCGCTAGTCCACTTTGTGGCGTTGGATCATCTGTCATG

Fig. 5. Genetic organization of the $p d x R-p d x S T$ intergenic region from C. glutamicum ATCC 13032. (a) Putative promoter sequences and candidate DNA-binding sites detected in the $89 \mathrm{bp} p d x R-p d x S T$ intergenic region. The mapped $5^{\prime}$ ends are marked (TSP) and the deduced -10 and -35 hexamers of the $p d x R$ and $p d x S T$ promoters are highlighted with grey boxes. The candidate DNA-binding sites (motifs 1-3) are shown and the location of an SOS box is indicated (Jochmann et al., 2009). The ATG start codons are shown in bold letters. (b) Comparative DNA sequence analysis of the $p d x R-p d x S T$ intergenic regions from actinobacteria. The DNA regions corresponding to the candidate DNA-binding sites from C. glutamicum ATCC 13032 (motifs 1-3) are boxed. An additional motif (motif 4) was detected in the genome sequence of $P$. acnes. Start codons are shown in bold type. Pa, P. acnes KPA171202; Jd, J. denitrificans DSM 20603; Ca, C. aurimucosum ATCC 700975; Cd, C. diphtheriae NCTC 13129; Cge, 'C. genitalium' ATCC 33030; Cm, C. matruchotii ATCC 14266; Cs, C. striatum ATCC 6940; Cgl, C. glutamicum ATCC 13032.

was investigated in vitro by DNA band shift assays with purified PdxR protein.

\section{DNA band shift assays with purified PdxR protein}

To demonstrate the specific binding of the regulatory protein PdxR to the detected sequence motifs in the $p d x R-$ $p d x S T$ intergenic region of the C. glutamicum genome, DNA band shift assays were performed (Fig. 6). The PdxR protein was tagged and purified by means of the IMPACT system, and the identity of the obtained protein was verified by MALDI-TOF MS (data not shown). The purified PdxR protein was used in DNA band shift assays together with a Cy3-labelled PCR fragment of $195 \mathrm{bp}$, containing the $89 \mathrm{bp} p d x R-p d x S T$ intergenic region. This amplified DNA fragment showed different electrophoretic mobilities following incubation with either the PdxR protein or the SOS response regulator LexA (Fig. 6a). This result confirmed that both C. glutamicum proteins interact directly with the $p d x R-p d x S T$ intergenic region. A genomic DNA fragment containing the DNA-binding site of the transcription regulator LtbR from C. glutamicum served as a negative control (Fig. 6a). The binding of PdxR to the $p d x R-p d x S T$ intergenic region was not prevented in vitro by the addition of either pyridoxal, PLP, pyridoxamine or pyridoxine to the DNA band shift assay, although the more highly shifted band was reduced in the presence of pyridoxamine or pyridoxine (Fig. 6a). Moreover, Cy3labelled, double-stranded 40-mers containing the sequence motifs 1-3 were applied in DNA band shift assays. Both PdxR and LexA were able to shift the specific 40-mer oligonucleotide containing motif 1 and the SOS box (Fig. $6 \mathrm{~b})$. When using a mutated version of motif 1 by introducing transitions into the $11 \mathrm{bp}$ sequence, the PdxR protein failed to shift the DNA fragment. On the other hand, transitions introduced into the genomic DNA regions flanking motif 1 did not affect the DNA binding of the PdxR protein (Fig. 6b). The PdxR protein also interacted with the 40 -mers containing motif 2 or motif 3 , but showed weaker interaction with these DNA fragments (Fig. 6c). As the three motifs are located very close to each other, the 40-mers representing motif 2 and motif 3 overlap with the adjacent motifs by a few bases. To clarify better the interaction of PdxR with motif 2 and motif 3 , the native flanking regions of these DNA stretches were replaced by transitions. In both cases, the PdxR protein showed a weak interaction with the respective 40mers, indicating that motif 2 and motif 3 can be recognized in vitro (Fig. 6c). These data demonstrated that the PdxR protein of C. glutamicum can interact in vitro with the three candidate DNA-binding sites that are located in the $p d x R-$ $p d x S T$ intergenic region.

Additional DNA band shift assays were performed with 40mer oligonucleotides containing motif 2 and motif 3 . As observed with the single DNA motifs, a weak interaction of PdxR was detectable (Fig. 6d). The orientation of these motifs within the 40-mer was subsequently altered in such a way that they were arranged as direct repeats. In both cases, the PdxR protein interacted with the 40-mers much better than with the DNA fragment containing the native orientation of the two motifs (Fig. 6d). This probably 
(a)

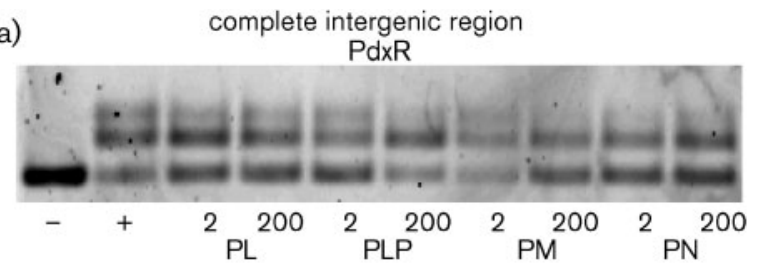

complete intergenic region

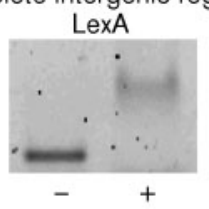

negative control

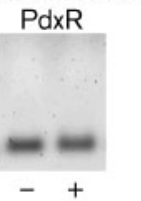

(b)

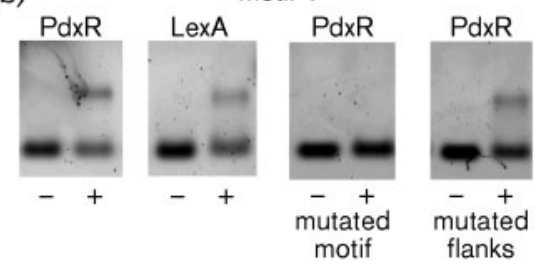

motif 1 GCTTCATCGTCCTCAAAAAAGTGGTCTAATGCAAGTGACT motif 1 * GCTTCATCGTCCTCAAAgggacaactCgATGCAAGTGACT flank 1* atcctgctacttctgggAAAGTGGTCTAgcatggacagtc

(c) motif 2

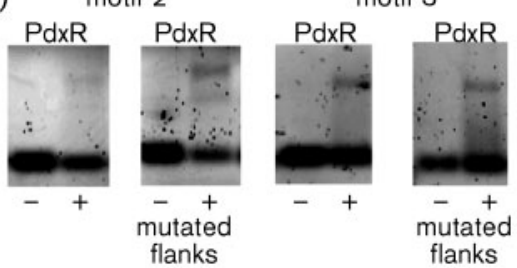

motif 2 GTCTAATGCAAGTGACTGAAAGTGGATCTACCGCTAGTCC

flank 2* actcggcatggacagtcaAAAGTGGATCTAttatcgactt motif 3 AGTGGATCTACCGCTAGTCCACTTTGTGGCGTTGGATCAT

flank $3^{*}$ gacaagctcgttatTAGTCCACTTTacaataccaagctgC

(d)

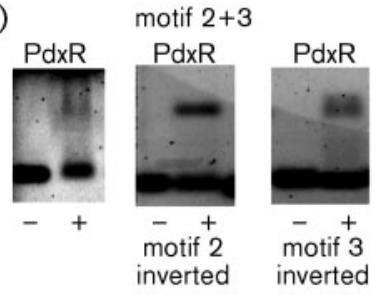

motif $2+3$ TGACTGAAAGTGGATCTACCGCTAGTCCACTTTGTGGCGT motif 2 inv TGACTG广TAGATCCACTTTCCG

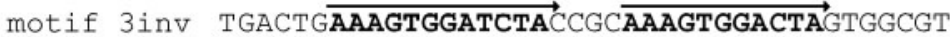

(e)

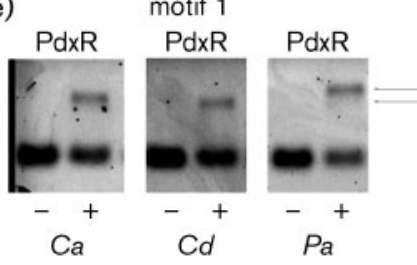

Fig. 6. DNA band shift assays performed with the purified PdxR protein from C. glutamicum ATCC 13032. (a) DNA band shift assays with a $195 \mathrm{bp}$ PCR product covering the $p d x R-p d x S T$ intergenic region. The DNA band shifts were performed with $\mathrm{PdxR}$ and LexA proteins (+). The first lanes of each assay show controls that do not contain purified protein (-). In the DNA band shift assays with PdxR, modified binding buffers were used, containing either 2 or $200 \mu \mathrm{M}$ of the vitamers pyridoxal (PL), pyridoxal 5'-phosphate (PLP), pyridoxamine (PM) or pyridoxine (PN). The LtbR binding-site from C. glutamicum ATCC 13032 served as a negative control (Brune et al., 2007). (b) DNA band shifts with oligonucleotides (40-mers) covering motif 1 . The DNA sequences of the 40-mers are shown. The sequence of motif 1 is shown in bold type. Mutations were introduced by transitions into either motif 1 (motif $1^{*}$ ) or the native flanking regions (flank $1^{*}$ ). The replaced bases are shown in lower-case letters. The SOS box is underlined. (c) DNA band shifts with 40-mers covering motif 2 or motif 3 . Mutations were introduced by transitions into the native flanking regions of motif 2 (flank $2^{*}$ ) and motif 3 (flank $3^{*}$ ). (d) DNA band shifts with 40-mers covering motif 2 and motif 3 . The 40-mers were modified by inverting either motif 2 (motif 2inv) or motif 3 (motif 3inv). The orientation of the motifs is indicated by arrows. (e) DNA band shift assays with 40-mers covering the motif 1 region from $C$. aurimucosum $(\mathrm{Ca}), \mathrm{C}$. diphtheriae $(\mathrm{Cd})$ and $P$. acnes $(\mathrm{Pa})$. Motif 1 and the additional motif 4 in $P$. acnes are arranged as direct repeats, indicated by arrows.

indicates that a direct repeat favours the interaction of PdxR with the DNA in vitro. Moreover, DNA band shift assays were carried out with 40-mer oligonucleotides containing motif 1 from C. aurimucosum, Corynebacterium diphtheriae and $P$. acnes to test whether the purified PdxR protein from C. glutamicum ATCC 13032 is able to interact also with 
these motifs present in the $p d x R-p d x S T$ intergenic region of other actinobacteria (Fig. 6e). In all cases, a clear retardation of the 40-mer DNA fragment was observed, further supporting the idea that the conserved $11 \mathrm{bp}$ region is relevant for the direct interaction of PdxR with the target DNA. The DNA band shift assay performed with a 40-mer containing motif 1 from $P$. acnes showed a different electrophoretic mobility when compared with the other assays (Fig. 6e). This difference may be due to the presence of an additional candidate DNA-binding site in this 40-mer sequence (motif 4) (Fig. 5b). By aligning the nucleotide sequences of the detected motifs, the DNA-binding site recognized by PdxR from C. glutamicum ATCC 13032 can be defined as AAAGTGGW $(-/$ T)CTA.

\section{DISCUSSION}

Our results provide evidence that the transcription regulator PdxR controls the biosynthesis of PLP in C. glutamicum by activating the expression of the PLP synthase genes $p d x S T$. Disruption of the $p d x R$ gene resulted in decreased expression of the $p d x S T$ genes and consequently in vitamin $\mathrm{B}_{6}$ auxotrophy. In an earlier study, growth assays performed with Streptomyces venezuelae ISP 5230 showed similar results, as disruption of the so-called ORF1 caused a growth requirement for pyridoxal (Magarvey et al., 2001). The corresponding gene region was isolated by complementing the $p d x-4$ mutation of $S$. venezuelae, indicating a relationship between the expression of ORF1 and the availability of pyridoxal. The protein encoded by ORF1 can be classified as a member of the MocR subfamily of GntR-type transcription regulators (Magarvey et al., 2001; Rigali et al., 2002). The PdxR protein from C. glutamicum was also grouped into this subfamily of regulatory proteins (Brune et al., 2005; McHardy et al., 2003). The GntR superfamily is one of the most abundant and widely distributed collections of metabolite-responsive transcription regulators in bacteria (Hoskisson \& Rigali, 2009). In principle, these regulators contain a conserved $\mathrm{N}$-terminal DNA-binding domain and a C-terminal effector-binding and/or oligomerization (E-b/ O) domain that show extensive variation, apparently reflecting the large diversity of effector molecules that they bind. Based on comprehensive amino acid sequence alignments of the E-b/O domains and secondary structural predictions, GntR-like transcription regulators were classified into seven subfamilies (Hoskisson \& Rigali, 2009). A characteristic feature of the MocR subfamily is the exceptional average length of the $\mathrm{E}-\mathrm{b} / \mathrm{O}$ domain that showed similarity to aspartate (class I) aminotransferases (Rigali et al., 2002). These PLP-dependent enzymes catalyse the reversible transfer of an amino group from an amino acid substrate to an $\alpha$-keto acid acceptor (Schneider et al., 2000). This sequence similarity raises the possibility that the E-b/O domains of MocR-type transcription regulators have catalytic activity (Rigali et al., 2002). However, the amino acid sequence alignment of PLP attachment sites from the actinobacterial PdxR-like proteins revealed the lack of the highly conserved lysine residue that is generally used for the covalent binding of the PLP group (Sigrist et al., 2010). Replacement of this essential lysine residue by serine, threonine or histidine may result in the loss of an enzymic function, which is consistent with a previous functional analysis in C. glutamicum, as aminotransferase activity was not detectable in enzyme assays with the purified PdxR protein (Marienhagen et al., 2005). This result is in marked contrast with observations made with the regulatory protein GabR from Bacillus subtilis, a MocRtype transcription regulator that positively controls the utilization of $\gamma$-aminobutyric acid and represses its own expression (Belitsky \& Sonenshein, 2002). The aminotransferase domain of GabR is probably essential for the proper functioning of this protein, as modification of the conserved lysine residue to alanine by site-directed mutagenesis led to a mutant form of the regulator that was unable to activate the expression of the divergently orientated gabTD operon, but retained the full ability to bind to the target DNA and to repress the gabR promoter (Belitsky \& Sonenshein, 2002). The atypical amino acid sequence of the PLP attachment site in the actinobacterial PdxR-like proteins implies moreover that the covalent binding of PLP to the E-b/O domain is dispensable for the regulatory activity of $\mathrm{PdxR}$, suggesting that the E-b/O domain has a different and hitherto unknown function in these proteins. Rigali et al. (2002) suggested that the E-b/O domain of MocR-type regulators may have been recruited to facilitate protein-protein interactions.

By using bioinformatic pattern searches, we detected three candidate DNA-binding sites for PdxR that are characterized by the consensus sequence AAAGTGGW $(-/ \mathrm{T}) \mathrm{CTA}$. The $11 \mathrm{bp}$ motifs 1 and 3 (AAAGTGGWCTA) and the 12 bp motif 2 (AAAGTGGATCTA) are apparently relevant for DNA binding of PdxR in vitro. Motif 1 and motif 2 represent a direct repeat that is separated by a spacer of $13 \mathrm{bp}$. Hence, these candidate DNA-binding sites are located on the same face of the DNA double helix. A similar genetic organization of candidate DNA-binding sites was detected in the nucleotide sequence linked to DNA binding of TauR, a MocR-type regulator that activates the expression of taurine utilization genes in Rhodobacter capsulatus (Wiethaus et al., 2008). The respective DNA region contains a $10 \mathrm{bp}$ direct repeat with the sequence CTGGAC(T/C)TAA and a 13 bp spacing (Wiethaus et al., 2008). Interestingly, the candidate DNAbinding sites of TauR and the $12 \mathrm{bp}$ motif 2 recognized by PdxR share the similar sequence TGGAYYTA, whereas the stretch of DNA sequence similarity to the 11 bp motif 1 is shorter (TGGWCY). DNA binding of the MocR-type regulator GabR was examined by DNase I footprinting and additional mutational studies of the recognized DNA region, revealing a $6 \mathrm{bp}$ direct repeat with the sequence ATACCA located within the minimal GabR-binding region (Belitsky, 2004). Accordingly, short, directly repeated DNA sequences are present in the candidate operators of the 
three MocR-type regulators. Another interesting aspect of MocR-type transcription regulators relates to their environmental sensing mechanism. The GabR protein from $B$. subtilis binds to its target DNA independently of PLP and $\gamma$-aminobutyric acid, but requires both metabolites for the activation of the gabTD operon (Belitsky \& Sonenshein, 2002). Disruption of the $p d x R$ gene in C. glutamicum resulted in decreased expression of the $p d x S T$ genes, which was also observed in the wild-type strain when supplementing the growth medium with either pyridoxal or PLP. How the PdxR regulator converts environmental information into the activation of $p d x S T$ gene expression is currently unknown and will be addressed in future studies.

\section{ACKNOWLEDGEMENTS}

We thank Alfred Pühler (Bielefeld University) for continuous scientific support and many helpful discussions. N. J. acknowledges the receipt of a scholarship from the International NRW Graduate School in Bioinformatics and Genome Research.

\section{REFERENCES}

Barreiro, C., Nakunst, D., Hüser, A. T., de Paz, H. D., Kalinowski, J. \& Martin, J. F. (2009). Microarray studies reveal a 'differential response' to moderate or severe heat shock of the HrcA- and HspR-dependent systems in Corynebacterium glutamicum. Microbiology 155, 359372.

Belitsky, B. R. (2004). Bacillus subtilis GabR, a protein with DNAbinding and aminotransferase domains, is a PLP-dependent transcriptional regulator. J Mol Biol 340, 655-664.

Belitsky, B. R. \& Sonenshein, A. L. (2002). GabR, a member of a novel protein family, regulates the utilization of gamma-aminobutyrate in Bacillus subtilis. Mol Microbiol 45, 569-583.

Brinkrolf, K., Brune, I. \& Tauch, A. (2007). The transcriptional regulatory network of the amino acid producer Corynebacterium glutamicum. J Biotechnol 129, 191-211.

Brune, I., Brinkrolf, K., Kalinowski, J., Pühler, A. \& Tauch, A. (2005). The individual and common repertoire of DNA-binding transcriptional regulators of Corynebacterium glutamicum, Corynebacterium efficiens, Corynebacterium diphtheriae and Corynebacterium jeikeium deduced from the complete genome sequences. BMC Genomics 6, 86.

Brune, I., Jochmann, N., Brinkrolf, K., Hüser, A. T., Gerstmeir, R., Eikmanns, B. J., Kalinowski, J., Pühler, A. \& Tauch, A. (2007). The IclR-type transcriptional repressor LtbR regulates the expression of leucine and tryptophan biosynthesis genes in the amino acid producer Corynebacterium glutamicum. J Bacteriol 189, 2720-2733.

Dsouza, M., Larsen, N. \& Overbeek, R. (1997). Searching for patterns in genomic data. Trends Genet 13, 497-498.

Eliot, A. C. \& Kirsch, J. F. (2004). Pyridoxal phosphate enzymes: mechanistic, structural, and evolutionary considerations. Annu Rev Biochem 73, 383-415.

Fitzpatrick, T. B., Amrhein, N., Kappes, B., Macheroux, P., Tews, I. \& Raschle, T. (2007). Two independent routes of de novo vitamin $\mathrm{B}_{6}$ biosynthesis: not that different after all. Biochem J 407, 1-13.

Gough, J., Karplus, K., Hughey, R. \& Chothia, C. (2001). Assignment of homology to genome sequences using a library of hidden Markov models that represent all proteins of known structure. J Mol Biol 313, 903-919.
Grant, S. G., Jessee, J., Bloom, F. R. \& Hanahan, D. (1990). Differential plasmid rescue from transgenic mouse DNAs into Escherichia coli methylation-restriction mutants. Proc Natl Acad Sci U S A 87, 4645-4649.

Horton, R. M., Hunt, H. D., Ho, S. N., Pullen, J. K. \& Pease, L. R. (1989). Engineering hybrid genes without the use of restriction enzymes: gene splicing by overlap extension. Gene 77, 61-68.

Hoskisson, P. A. \& Rigali, S. (2009). Chapter 1: variation in form and function of the helix-turn-helix regulators of the GntR superfamily. Adv Appl Microbiol 69, 1-22.

Ikeda, M. \& Nakagawa, S. (2003). The Corynebacterium glutamicum genome: features and impacts on biotechnological processes. Appl Microbiol Biotechnol 62, 99-109.

Jochmann, N., Kurze, A. K., Czaja, L. F., Brinkrolf, K., Brune, I., Hüser, A. T., Hansmeier, N., Pühler, A., Borovok, I. \& Tauch, A. (2009). Genetic makeup of the Corynebacterium glutamicum LexA regulon deduced from comparative transcriptomics and in vitro DNA band shift assays. Microbiology 155, 1459-1477.

Kalinowski, J., Bathe, B., Bartels, D., Bischoff, N., Bott, M., Burkovski, A., Dusch, N., Eggeling, L., Eikmanns, B. J. \& other authors (2003). The complete Corynebacterium glutamicum ATCC 13032 genome sequence and its impact on the production of $\mathrm{L}^{-}$ aspartate-derived amino acids and vitamins. J Biotechnol 104, 5-25.

Keilhauer, C., Eggeling, L. \& Sahm, H. (1993). Isoleucine synthesis in Corynebacterium glutamicum: molecular analysis of the ilvB-ilvN-ilvC operon. J Bacteriol 175, 5595-5603.

Kirchner, O. \& Tauch, A. (2003). Tools for genetic engineering in the amino acid-producing bacterium Corynebacterium glutamicum. J Biotechnol 104, 287-299.

Kohl, T. A. \& Tauch, A. (2009). The GlxR regulon of the amino acid producer Corynebacterium glutamicum: detection of the corynebacterial core regulon and integration into the transcriptional regulatory network model. J Biotechnol 143, 239-246.

Kurtz, S., Choudhuri, J. V., Ohlebusch, E., Schleiermacher, C., Stoye, J. \& Giegerich, R. (2001). REPuter: the manifold applications of repeat analysis on a genomic scale. Nucleic Acids Res 29, 4633-4642.

Larkin, M. A., Blackshields, G., Brown, N. P., Chenna, R., McGettigan, P. A., McWilliam, H., Valentin, F., Wallace, I. M., Wilm, A. \& other authors (2007). CLUSTAL W and CLUSTAL_X version 2.0. Bioinformatics 23, 2947-2948.

Magarvey, N., He, J., Aidoo, K. A. \& Vining, L. C. (2001). The $p d x$ genetic marker adjacent to the chloramphenicol biosynthesis gene cluster in Streptomyces venezuelae ISP5230: functional characterization. Microbiology 147, 2103-2112.

Marchler-Bauer, A., Anderson, J. B., Chitsaz, F., Derbyshire, M. K., DeWeese-Scott, C., Fong, J. H., Geer, L. Y., Geer, R. C., Gonzales, N. R. \& other authors (2009). CDD: specific functional annotation with the Conserved Domain Database. Nucleic Acids Res 37, D205D210.

Marienhagen, J., Kennerknecht, N., Sahm, H. \& Eggeling, L. (2005). Functional analysis of all aminotransferase proteins inferred from the genome sequence of Corynebacterium glutamicum. J Bacteriol 187, 7639-7646.

Markowitz, V. M., Korzeniewski, F., Palaniappan, K., Szeto, E., Werner, G., Padki, A., Zhao, X., Dubchak, I., Hugenholtz, P. \& other authors (2006). The integrated microbial genomes (IMG) system. Nucleic Acids Res 34, D344-D348.

McHardy, A. C., Tauch, A., Rückert, C., Pühler, A. \& Kalinowski, J. (2003). Genome-based analysis of biosynthetic aminotransferase genes of Corynebacterium glutamicum. J Biotechnol 104, 229-240.

Mormann, S., Lömker, A., Rückert, C., Gaigalat, L., Tauch, A., Pühler, A. \& Kalinowski, J. (2006). Random mutagenesis in Corynebacterium 
glutamicum ATCC 13032 using an IS6100-based transposon vector identified the last unknown gene in the histidine biosynthesis pathway. BMC Genomics 7, 205.

Pátek, M., Nesvera, J., Guyonvarch, A., Reyes, O. \& Leblon, G. (2003). Promoters of Corynebacterium glutamicum. J Biotechnol 104, 311-323.

Rigali, S., Derouaux, A., Giannotta, F. \& Dusart, J. (2002). Subdivision of the helix-turn-helix GntR family of bacterial regulators in the FadR, HutC, MocR, and YtrA subfamilies. J Biol Chem 277, 12507-12515.

Rodionov, D. A. (2007). Comparative genomic reconstruction of transcriptional regulatory networks in bacteria. Chem Rev 107, 3467-3497.

Sambrook, J., Fritsch, E. F. \& Maniatis, T. (1989). Molecular Cloning: a Laboratory Manual, 2nd edn. Cold Spring Harbor, NY: Cold Spring Harbor Laboratory.

Schäfer, A., Tauch, A., Jäger, W., Kalinowski, J., Thierbach, G. \& Pühler, A. (1994). Small mobilizable multi-purpose cloning vectors derived from the Escherichia coli plasmids pK18 and pK19: selection of defined deletions in the chromosome of Corynebacterium glutamicum. Gene 145, 69-73.

Schneider, G., Kack, H. \& Lindqvist, Y. (2000). The manifold of vitamin $\mathrm{B}_{6}$ dependent enzymes. Structure 8, R1-R6.

Sigrist, C. J., Cerutti, L., de Castro, E., Langendijk-Genevaux, P. S., Bulliard, V., Bairoch, A. \& Hulo, N. (2010). PROSITE, a protein domain database for functional characterization and annotation. Nucleic Acids Res 38, D161-D166.
Strohmeier, M., Raschle, T., Mazurkiewicz, J., Rippe, K., Sinning, I., Fitzpatrick, T. B. \& Tews, I. (2006). Structure of a bacterial pyridoxal 5 '-phosphate synthase complex. Proc Natl Acad Sci U S A 103, 1928419289.

Tanaka, T., Tateno, Y. \& Gojobori, T. (2005). Evolution of vitamin $\mathrm{B}_{6}$ (pyridoxine) metabolism by gain and loss of genes. Mol Biol Evol 22, 243-250.

Tauch, A., Kirchner, O., Wehmeier, L., Kalinowski, J. \& Pühler, A. (1994). Corynebacterium glutamicum DNA is subjected to methylation-restriction in Escherichia coli. FEMS Microbiol Lett 123, 343347.

Tauch, A., Kassing, F., Kalinowski, J. \& Pühler, A. (1995). The Corynebacterium xerosis composite transposon Tn5432 consists of two identical insertion sequences, designated IS1249, flanking the erythromycin resistance gene ermCX. Plasmid 34, 119-131.

Tauch, A., Kirchner, O., Löffler, B., Götker, S., Pühler, A. \& Kalinowski, J. (2002). Efficient electrotransformation of Corynebacterium diphtheriae with a mini-replicon derived from the Corynebacterium glutamicum plasmid pGA1. Curr Microbiol 45, 362-367.

Wiethaus, J., Schubert, B., Pfänder, Y., Narberhaus, F. \& Masepohl, B. (2008). The GntR-like regulator TauR activates expression of taurine utilization genes in Rhodobacter capsulatus. J Bacteriol 190, 487-493.

Edited by: C. W. Price 